\title{
An Internal Standard High-Performance Liquid Chromatography Method for Simultaneous Quantification of Candesartan and Hydrochlorothiazide in Combined Formulations
}

\author{
FAISAL K. ALGETHAMI \\ Department of Chemistry, College of Science, Imam Mohammad Ibn Saud Islamic \\ University (IMSIU), Riyadh 11432, Saudi Arabia. \\ *Corresponding author E-mail: falgethami@imamu.edu.sa \\ http://dx.doi.org/10.13005/ojc/370509
}

(Received: July 15, 2021; Accepted: September 16, 2021)

\begin{abstract}
The internal standard method is a versatile procedure that avoids misleading results caused by the instability of the chromatographic system or inexperienced workers. It is an effective way to judge the accuracy of any obtained data. As the detector responses of chlorzoxazone (CZN) resemble those of candesartan (CDZN) and hydrochlorothiazide (HCTZ), CZN was employed as an internal standard. Herein, a simple chromatographic method was established for quantification of CDZN and HCTZ. Isocratic elution was conducted using $1 \%$ premixed acetonitrile/formic acid $(7: 3 \mathrm{v} / \mathrm{v})$ at a $0.8 \mathrm{~mL} / \mathrm{min}$ flowrate. The separation of the three components was maintained using the universal $20 \mu \mathrm{L}$ loop, and for further simplicity in application, the analysis was optimized at $25^{\circ} \mathrm{C}$. CDZN, $\mathrm{HCTZ}$, and CZN were simultaneously monitored and quantified at $270 \mathrm{~nm}$. The method developed here complies with all the validation limits according to the British Pharmacopoeia (BP), United States Pharmacopoeia (USP), and the guidelines of the International Council For Harmonisation (ICH). The method proved to be linear in the range of 6.4 to $25.6 \mu \mathrm{g} / \mathrm{mL}$ and $5.0-20 \mu \mathrm{g} / \mathrm{mL}$ for CDZN and $\mathrm{HCTZ}$, respectively, while the quantitation detection limits were less than $1.0 \mu \mathrm{g} / \mathrm{mL}$ for both.
\end{abstract}

Keywords: HPLC, Internal standard, Candesartan, Hydrochlorothiazide, Chlorzoxazone, Isocratic.

\section{INTRODUCTION}

Candesartan (1-hydroxyethyl 2-ethoxy$1-[p-(o-1 \mathrm{H}$-tetrazole-5-yl phenyl)benzyl $]$ -7-benzimidazole carboxylate, cyclohexyl carbonate) (CDZN) and hydrochlorothiazide (6-chloro-3,4dihydro-2H-1,2,4-benzothiadiazine-7-sulphonamide 1,1-dioxide) (HCTZ) are diuretics and angiotensin II receptor blockers prescribed for hypertension
(Fig. 1) ${ }^{1-3}$ Currently, researchers are interested in developing assay methods to validate their effectiveness, and though several have been developed for HCTZ and CDZN, most tests for these compounds separately, and only a few examine both substances simultaneously. Some researchers use spectrophotometric methods because of their ease of use $e^{4,5}$, whereas others use LC-MS/MS, which would be ideal if not for

This is an Open Access article licensed under a Creative Commons license: Attribution 4.0 International (CC- BY). Published by Oriental Scientific Publishing Company @ 2018 
the high cost of the equipment ${ }^{3}$. High-performance liquid chromatography-ultraviolet (HPLC-UV) can be used in place of spectrophotometric procedures as it includes separation, and it is more affordable than the LC-MS/MS. Based on these reasons, an increasing volume of work has explored the use of HPLC-UV in assaying pharmaceutical formulations ${ }^{6-11}$.

Method validation is a valuable topic in quantitative analysis because it is essential for demonstrating the reliability of any innovative analytical methodology $y^{12,13}$. Because of the importance of this process, many agencies have set up a standard procedure ${ }^{14-16}$. Method validation is an exclusive protocol that tests the accuracy, specificity, precision, reproducibility, limit of detection (LOD), and limit of quantification (LOQ) of a method to establish its suitability for a given purpose ${ }^{17}$. The internal standard method is a powerful technique avoids the generation of misleading results caused by the detector or separation system ${ }^{18}$.

This research was aimed at developing an HPLC photodiode array (HPLC-PDA) for simultaneously quantifying CDZN and HCTZ in pharmaceutical formulations. The muscle relaxant chlorozoxazone (5-chloro-3H-1,3-benzoxazole-2-one) ${ }^{19}$ (CZN) presented in Fig. 1c was used as an internal standard substrate to ensure the production of reliable results. As a result, this study presents a simple and fast analytical approach for application in quality control and research analysis.

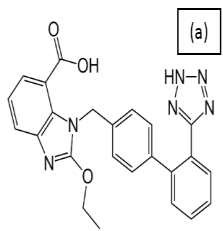

$$
\text { (b) }
$$

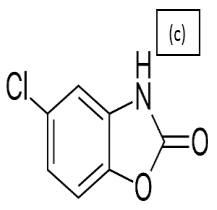

Fig. 1. The chemical structure of (a) candezartan, (b) hydrochlorothiazide, and (c) chlorozoxazone

\section{EXPERIMENTAL}

\section{Chemicals}

Working standards of CDZN, HCTZ, and CZN active pharmaceutical ingredients were supplied from Ranbaxy Laboratories Limited, India. The combined tablets containing $16 \mathrm{mg}$ CDZN and $12.5 \mathrm{mg} \mathrm{HCTZ}$ were purchased from a local market in Riyadh, KSA. CZN is an analgesic that was employed as an internal standard. HPLC-grade solvents acetonitrile was purchased from Sharlau, Spain. The placebo of the drug formulation was purchased from the Tabuk factory for pharmaceutical industries in Riyadh.

\section{Preparation of Solutions}

For preparation of the mobile phase solution, $700 \mathrm{~mL}$ acetonitrile and $300 \mathrm{~mL}$ formic acid were combined, and the solution was vacuum filtered, sonicated for $30 \mathrm{~min}$, and cooled to $25^{\circ} \mathrm{C}$. CDZN $(0.16 \mathrm{~g})$ and $\mathrm{HCTZ}(0.125 \mathrm{~g})$ were transferred to a $100 \mathrm{~mL}$ volumetric flask, which was half-filled with the mobile phase solution, sonicated for $30 \mathrm{~min}$, and cooled to $25^{\circ} \mathrm{C}$. It was then filled to the mark with the same solvent. The CZN (300 mg/L) to be utilized as an internal standard substrate was prepared in the same manner. The appropriate stock solution volume was mixed with an aliquot of CZN stock solution, then diluted to $100 \mathrm{~mL}$ to attain a concentration of $16 \mu \mathrm{g} / \mathrm{mL} \mathrm{CDZN}, 12.5 \mu \mathrm{g} / \mathrm{mL} \mathrm{HCTZ}$, and $30 \mu \mathrm{g} /$ $\mathrm{mL} C Z N$. The average weight of 20 tablets $(0.2596$ g) underwent similar procedures to prepare a stock. Subsequent dilution was carried out, and the appropriate volume of CZN was added prior to the solution being adjusted to the appropriate final volume. Placebo pow-der $(0.2311 \mathrm{~g})$ was transferred to a $100 \mathrm{~mL}$ volumetric flask and processed similarly for sample preparation.

A $0.22 \mu \mathrm{m}$ nylon filter, which does not affect the concentration of solutions, was employed to filter solutions prior to their injection into the Shimadzu autosampler.

\section{Chromatographic Conditions and Method Validation}

The validation was conducted utilizing an HPLC/UV-Vis (Prominence, Shimadzu, Japan). Several columns were tested, including phenylhexyl, octyl (C8), octadecyl (C18). To determine the best elution, the mobile phase composition was varied in terms of the formic acid/acetonitrile ratio. Furthermore, column temperatures ranging $20-40^{\circ} \mathrm{C}$ were tested, as were flowrates ranging 0.5-2.0 mL/minute. CDZN, HCTZ, and CZN were moni-tored at $270 \mathrm{~nm}$. 
The previously described standard solution was employed as a test for system suitability. The linearity and accuracy tests were performed using serial concentrations in the range of $40-160 \%$ of the target concentration. Typically, CDZN concentrations were $6.4,9.6,12.8,16,19.2,22.4$, and $25.6 \mu \mathrm{g} /$ $\mathrm{mL}$, while $5.0,7.5,10,12.5,15,17.5$, and 20 $\mu \mathrm{g} / \mathrm{mL}$ concentrations were used for HCTZ. The solutions for linearity tests were prepared as a mixed standard, and each was injected with an internal standard aliquot to a final concentration of $30 \mu \mathrm{g} / \mathrm{mL} \mathrm{CZN}$. The detection and quantification limits were calculated statistically from the linearity results ${ }^{16}$. The standard, sample, and placebo solutions at optimized chromatographic conditions were used to investigate the procedure selectivity. Synthetic samples with 80 and $120 \%$ of the target concentrations were employed to test for robustness through intraday and interday studies. The three solutions were analyzed five times on the same day and on three consecutive days for the interday determination. The method robustness was tested by examining the effects resulting from slight alterations to the optimized chromatographic conditions. The detection wavelength varied by $\pm 5 \mathrm{~nm}$; the mobile phase composition was altered by $\pm 5 \%$, and the column temperature was varied by $\pm 5^{\circ} \mathrm{C}$. Each parameter was changed separately, the analysis was conducted, and the recovery percentage then calculated.

\section{RESULTS}

The separation of CDZN, HCTZ, and the internal standard CZN was accomplished using a mobile phase consisting of acetonitrile/formic acid
$(7: 3 \mathrm{v} / \mathrm{v})$ with a $\mathrm{pH}$ of 2.8 . A flowrate of $0.8 \mathrm{~mL} / \mathrm{min}$ was the best within the tested range, and the phenylhexyl column performed better than $\mathrm{C} 8$ and $\mathrm{C} 18$ in separating this combination. Furthermore, the PDA detector revealed that the best response for the three components was at $270 \mathrm{~nm}$. Moreover, the commonly used $20 \mu \mathrm{L}$ loop volume was selected to ease the procedure's applicability.

To validate this method, the USP protocol was followed. A system suitability test that used a mixed standard solution of the target concentration was conducted. As monitored in Table 1, the statistical parameters of CDZN and HCTZ were within the acceptance criteria, implying the method's repeatability.

The area of the analytes was divided by that of the internal standard to be corrected. Fig. 2 illustrates the regression lines driven from the corrected peak area versus the concentration plot. The results show that the method was linear within the examined concentrations, and the correlation coefficient for both pharmaceutical ingredients was 0.999 .

Detection and quantitation limits were determined and considered as an integral part of the validation protocol. The LOD and LOQ were statistically calculated using the results of linear regressions via Equations (1) and (2), respectively.

$$
\begin{aligned}
& \mathrm{LOD}=3^{\star} \mathrm{RMSE} \\
& \mathrm{LOD}=10^{\star} \mathrm{RMSE}
\end{aligned}
$$

\begin{tabular}{|c|c|c|c|c|c|}
\hline & HCTZ & $\mathrm{CDZN}$ & $\mathrm{CZN}$ & HCTZ (corrected) & CANDI (corrected) \\
\hline STD 1 & $1,284,626$ & 146,064 & $2,947,909$ & 0.435775 & 0.049548 \\
\hline STD 2 & $1,274,131$ & 146,875 & $2,948,752$ & 0.432092 & 0.049809 \\
\hline STD 3 & $1,279,409$ & 147,677 & $2,948,303$ & 0.433948 & 0.050089 \\
\hline STD 4 & $1,277,206$ & 147,013 & $2,948,774$ & 0.433131 & 0.049856 \\
\hline STD 5 & $1,279,045$ & 147,166 & $2,948,493$ & 0.433796 & 0.049912 \\
\hline STD 6 & $1,278,067$ & 148,323 & $2,948,280$ & 0.433496 & 0.050308 \\
\hline Avg. & $1,278,747$ & $147,186.3$ & $2,948,419$ & 0.433706 & 0.04992 \\
\hline STDV & 3441.925 & 764.1706 & 327.1897 & 0.001211 & 0.000258 \\
\hline RSD & 0.269164 & 0.519186 & 0.011097 & 0.279161 & 0.517656 \\
\hline Avg. tailing factor & 1.425 & 1.658 & 1.367 & & \\
\hline Avg. resolution & 8.936 & 4.847 & 4.947 & & \\
\hline Avg. theoretical plates number & 5957.6 & 8014.6 & $10,777.8$ & & \\
\hline
\end{tabular}



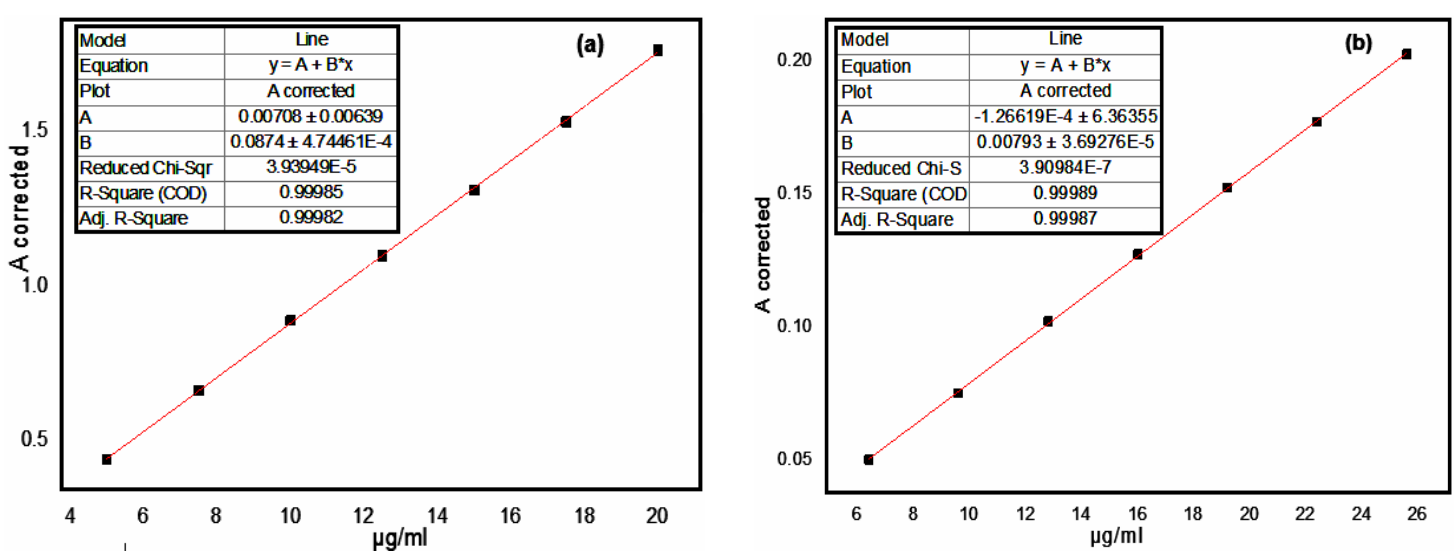

Fig. 2. The linearity relationships of the concentrations and their corrected area for (a) hydrochlorothiazide and (b) candesartan

The root mean square error (RMSE) was driven using the LINEST function (Microsoft Excel 2019): 0.0064 and 0.0006 for CDZN and HCTZ, respectively. For CDZN, the LOD was found to be $1.9 \times 10-2 \mu \mathrm{g} / \mathrm{mL}$, while the LOQ was $6.4 \times 10-2$ $\mu \mathrm{g} / \mathrm{mL}$. On the other hand, the values for HCTZ were $0.2 \times 10-2$ and $0.6 \times 10-2 \mu \mathrm{g} / \mathrm{mL}$, respectively.

The selectivity of the procedure was examined using the standard, sample, and placebo solutions in which the sample and standard solutions were injected with the internal standard. The results are shown in Fig. 3. Fig. 3b,c show that the excipients caused no alteration to the retention time of the active ingredients or the internal standard. Fig. 3a shows two peaks with a negligible area at the retention time of $\mathrm{HCTZ}$ and CZN. These peaks could be attributed to cross-contamination from samples or standard injections. These results were considered acceptable since the areas were insignificant compared to the sample peak areas ${ }^{14,16}$.
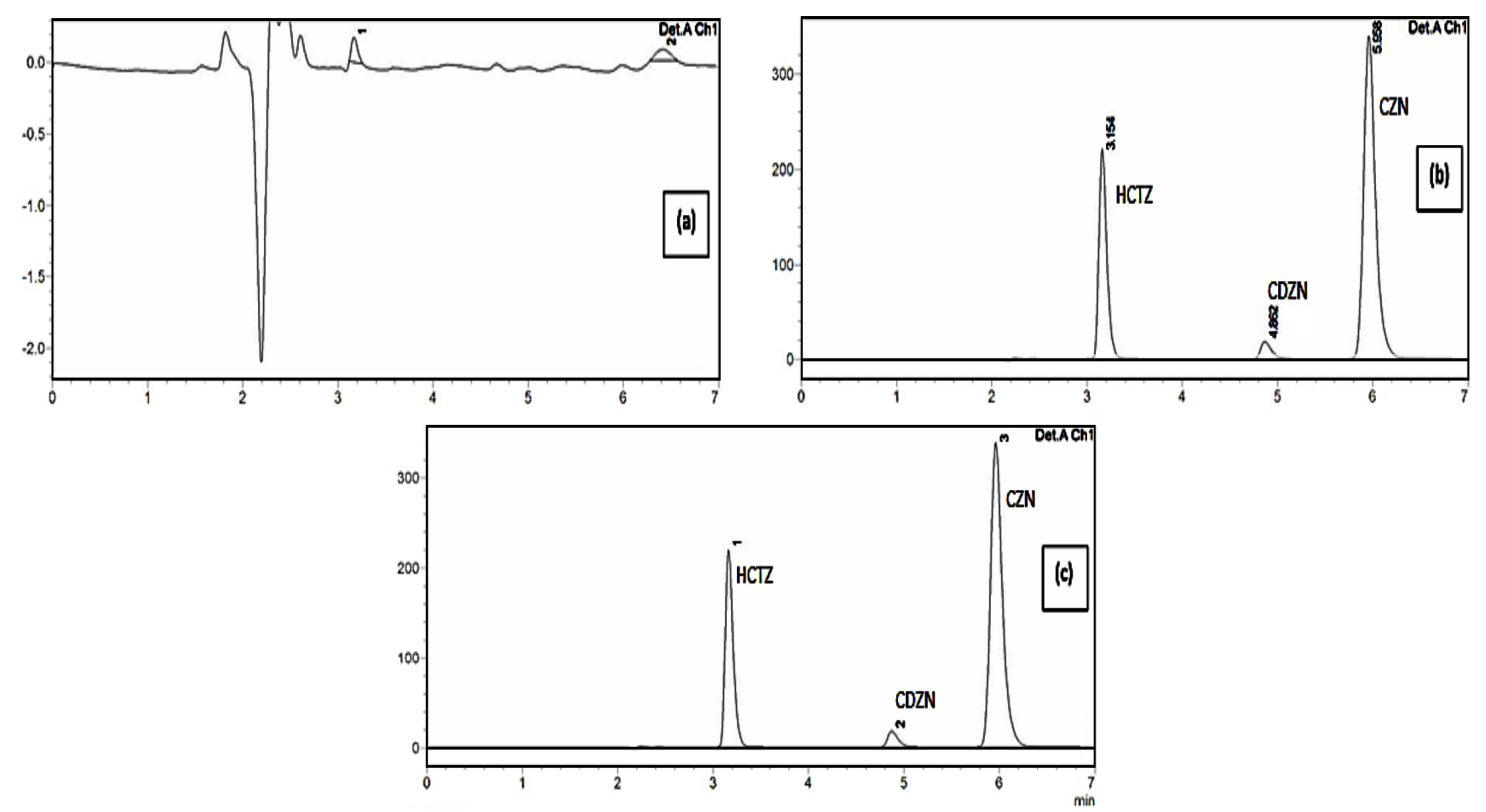

Fig. 3. Selectivity test using the placebo, the standard solution at target concentration, and the sample at target concentration injected with internal standard

Furthermore, the accuracy of the method was investigated, revealing excellent consistency in the recovery values for a concentration range of
$80-120 \%$ for both drugs. The average recovery for HCTZ and CDZN was 99.6 and $100.1 \%$, respectively. The RSD values for these recoveries 
ranged between 0.8 and 1.6, showing that the results for both drugs were within the acceptance criteria (RSD $\leq 2 \%$ ). These findings were comparable to, or better than, some results found in the literature for earlier methods ${ }^{18-20}$.

Moreover, the precision study was performed using the solutions prepared for the accuracy test. The repeatability of the internal standard method was inspected by conducting five consecutive assays for the test solutions. The procedure showed assay results in the range of 98.7-101.5 and 98.2-100.7 for HCTZ and CDZN, respectively. It is worth mentioning that the RSD values of the intraday investigations were within the acceptance criteria for both drugs and that the reproducibility of the procedure was tested by conducting the assay test on three different days. The results consistently reflected the method's reliability Table $2^{16,20}$.

Table 2: Precisions results for hydrochlorothiazide and candesartan using chlorzoxazone as an internal standard

\begin{tabular}{lcccccc}
\hline \multicolumn{5}{c}{ HCTZ } & \multicolumn{5}{c}{ CDZN } \\
Repeatability & & & & & & \\
\hline Assay No. & $80 \%$ & $100 \%$ & $120 \%$ & $80 \%$ & $100 \%$ & $120 \%$ \\
1 & 101.5 & 100.4 & 100.8 & 98.9 & 99.4 & 99 \\
2 & 98.7 & 99.3 & 100.2 & 98.4 & 99.1 & 99.8 \\
3 & 101.6 & 100.6 & 99.4 & 98.2 & 100.6 & 99 \\
4 & 101.5 & 99.2 & 99.1 & 100.2 & 98.4 & 98.2 \\
5 & 101.7 & 99.8 & 99.6 & 99.5 & 100.7 & 99.1 \\
Average & 101 & 99.86 & 99.82 & 99.04 & 99.64 & 99.02 \\
\%RSD & 1.3 & 0.6 & 0.7 & 0.8 & 1.0 & 0.6 \\
Reproducibility & & & & & & \\
1 & 100.8 & 100 & 100.8 & 99 & 99.1 & 100.0 \\
2 & 100.4 & 101 & 100.4 & 100 & 101.0 & 101.0 \\
3 & 100.9 & 100 & 100.2 & 99 & 100.0 & 99.0 \\
Average & 100.7 & 100.3 & 100.5 & 99.3 & 100.0 & 100.0 \\
\%RSD & 0.3 & 0.6 & 0.3 & 0.6 & 1.0 & 1.0 \\
\hline
\end{tabular}

The robustness of the internal standard method was investigated by changing various parameters of the optimized conditions separately. The method performance was relatively consistent even during the slight changes of the chromatographic parameters, as shown in Table 3. The RSD value for the assay at the optimized and altered parameters was less than $2 \%$. In addition, the average assay results of 99.7 and 100.8 for HCTZ and CDZN, respectively, demonstrate the robustness of this method.
Table 3: Test of method robustness based on measurement of HCTZ and CDZN using the developed internal standard method under optimized and altered chromatographic conditions

\begin{tabular}{llcc}
\hline $\begin{array}{l}\text { No } \\
\text { Recovery \% } \\
\text { Recovery \% }\end{array}$ & CONDITION & HCTZ & \\
\hline 1 & & & \\
\hline & Optimized conditions & 99.3 & 100.2 \\
3 & Mor 5 degree Celsius & 100.2 & 101.9 \\
4 & less 5 degree Celsius & 100.1 & 100.5 \\
5 & 5\% More flow rate & 99.4 & 99.7 \\
6 & 5\% less flow rate & 99.0 & 102.9 \\
7 & 5\% more Organic solvent & 100.2 & 102.0 \\
8 & 5\% less Organic solvent & 99.5 & 99.3 \\
9 & More 3 nm & 99.8 & 100.4 \\
& Less 3 nm & 99.8 & 100.5 \\
& Avg & 99.7 & 100.8 \\
& $\%$ RSD & 0.428 & 1.2 \\
\hline
\end{tabular}

Assay of local-market tablets by the developed method

The labeled content of HCTZ and CDZN in the tablet was 16 and $12.5 \mathrm{mg}$, respectively. The sample solution was injected with the internal standard (CZN), completed to $50 \mathrm{~mL}$ with the same solvent, and analyzed at optimized chromatographic conditions. The content of the HCTZ and CDZN tablets was determined to be within the acceptable range with an assay of 100.2 and $103.7 \%$, respectively.

\section{CONCLUSION}

A reversed-phase HPLC-PDA procedure was developed for the simultaneous quantification of HCTZ and CDZN combined in pharmaceutical formulations in which CZN was employed as an internal standard. Compared to conventional HPLC methods, the method developed here has the advantage of allowing self-correction via inclusion of the internal standard. The validated internal standard method successfully bypassed all the obstacles associated with fluctuations in the detector, temperature, pump flow, and mobile phase composition, thereby showing great potential for application in routine analysis and research.

\section{ACKNOWLEDGMENT}

This research did not receive any specific grant from funding agencies in the public, commercial, or not-for-profit sectors.

\section{Conflict of Interest}

The author declares that he has no conflict of interest. 


\section{REFERENCES}

1. Khedr, A. E. Simultaneous Determination of Candesartan Cilexetil and Hydrochlorothiazide by High-Performance Liquid Chromatography. J. King Abdulaziz Univ. Med Sci., 2008, 15, 3-13.

2. Srikalyani, V.; Madhuri, T.; Sareesh, K.; Kumar, R.M. Simultaneous estimation of hydrochlorthiazide and candesartan cilextil in bulk and pharmaceutical dosage forms by RP-HPLC PDA method. Int. J. Pharm. Sci. Res., 2018, 17, 150-157.

3. Stolarczyk, M.; Maślanka, A.; Apola, A.; Kwiecień, A.; Hubicka, U. Determination of candesartan, hydrochlorothiazide and rosuvastatin s active ingredients of a polytablet by a chromatographic-densitometric method. Acta Pol. Pharm., 2020, 77, 833-840.

4. Marghany, K.A.; Salamorcid, R.A.; Hadadorcid, G.M.; Ibrahim, E. Green UV Absorbance Ratio Method for Determination of Eprosartan and Hydrochlorothiazide. Rec. Pharm. Biomed. Sci., 2021, 5, 90-99.

5. Grewal, I.K.; Singh, S.; Arora, S.; Sharma, N. Development and validation of UVspectrophotometer analytical method of eflornithine hydrochloride. Plant Arch., 2020, 20, 3265-3270.

6. el Karbane, M.; Benchekroun, Y.H.; Abousalih, F.Z.; Bennani, I.; Azougagh, M.; Saffaj, T.; Bouatia, M. Development and val-idation of a UPLC-DAD method for the simultaneous quantification of eight antihypertensive drugs in the pharmaceutical matrix. J. AOAC Int., 2020, 104, 562-570.

7. Patel, M.; Kothari, C. Quantitative bioanalysis of pitavastatin and candesartan in rat plasma by HPLC-UV: Assessment of pharmacokinetic drug-drug interaction. $J$. Chromatogr. B., 2020, 1138, 121962.

8. Tan, A.; Gui, X.; Wong, M.; Deng, H.; Gu, G.; Fanaras, C.; Fanaras, J.C. Simultaneous quantification of candesartan and irbesartan in rabbit eye tissues by liquid chromatographytandem mass spectrometry. Biomed. Chromatogr., 2020, 34, e4808.

9. Bulk, A.I. Int. J. Mod. Pharm. Research., 2020, 11,6 .

10. Marghany, K.A.; Abdelsalam, R.A.; Haddad,
G.M. HPLC method transfer study for simultaneous determination of seven angiotensin II receptor blockers. J. Sep. Sci., 2020, 43, 1398-1405.

11. Janet, B.S.; Suthakaran, R.; Kymar, B.P.; Chankar, C.H.; Syed, G.; Suneetha, K. A Prospective Validation of Hydrocholoro-thiazide and Bisoprolol Fumerate in its Pure and Bulk Dosage Forms by using RP-HPLC Technique. Asian J. Pharm. Anal., 2020, 10, 1-6.

12. Hopfgartner, G. Bioanalytical Method Validation: How Much Should We Do and How Should We Document?; Springer: Berlin/Heidelberg, Germany., 2020.

13. Ozkan, S.A. Analytical method validation: The importance for pharmaceutical analysis. Pharm. Sci., 2018, 24, 1-2.

14. Q2 (R1) validation of analytical procedures. In ICH Quality Guidelines; Teasdale, A., Elder, D., Nims, R.W., Eds.; Wiley: Hoboken, NY, USA, 2017, 127-166.

15. Pharmacopeia. General Chapters: < 1225> Validation of Compendial Procedures., 2011, 778-782.

16. $\mathrm{ICH} . \mathrm{Q} 2(\mathrm{R} 1)$ : Validation of analytical procedures: Text and methodology. In International Conference on Harmonization; ICH: Geneva, Switzerland., 2005.

17. Raposo, F.; Ibelli-Bianco, C. Performance parameters for analytical method validation: Controversies and discrepancies among numerous guidelines. TrAC Trends Anal. Chem., 2020, 129, 115913.

18. Gülfen, M.; Canbaz, Y.; Özdemir, A. Simultaneous Determination of Amoxicillin, Lansoprazole, and Levofloxacin in Pharmaceuticals by HPLC with UV-Vis Detector. J. Anal. Test., 2020, 4, 45-53.

19. Roy, P.; Ghosh, A.J.C. Mechanochemical cocrystallization to improve the physicochemical properties of chlorzoxazone. Cryst Eng Comm., 2020, 22, 4611-4620.

20. Puumalainen, I.; Halonen, P.; Johnson, K.; Enlund, H.; Marja Validation of the United States Pharmacopeia (USP) medication counselling behaviour guidelines. Airaksinen Pharm. Educ., 2005, 5, 87-96. 